\title{
Análisis comparativo entre un modelo clásico docente y un ciclo de mejora docente mediante resolución de problemas aplicado a la asignatura de Prehistoria I (Grado en Historia)
}

\section{Comparative analysis between a Classic Teaching Model and a Cycle of Teaching Improvement through Problem Resolution applied to the Subject of Prehistory I (Degree in History)}

Álvaro Gómez Peña

ORCID: https://orcid.org/0000-0002-2926-5243

Universidad de Sevilla

Departamento de Prehistoria y Arqueología

agomez19@us.es

DOI: http://dx.doi.org/10.12795/9788447231003.091

Pp.: 1964-1985 


\section{Descripción del contexto}

En el presente texto se analiza el ciclo de innovación docente llevado a cabo durante el curso 2020/2021, inspirado en las recomendaciones publicadas por Porlán (2017), dentro de la asignatura Prehistoria I: las sociedades cazadoras y recolectoras. Se trata de una asignatura obligatoria impartida en el primer cuatrimestre del primer curso del Grado en Historia. En concreto, dicho proyecto se ha aplicado al Grupo 3, cuyo horario de clases se ha distribuido de lunes a jueves en horario de 16 a 17h.

Por lo que respecta a mi docencia, este curso académico ha sido el sexto año que he impartido esta asignatura. Gracias a ello, empiezo a conocer sus contenidos bastante bien, así como su bibliografía específica asociada y las novedades que ocurren en esta área de estudio año tras año. No obstante, esto último sigue generándome un especial estrés porque, además de tener que enseñar unos contenidos que no se ajustan a mi área de especialización investigadora, tengo que estar continuamente reciclándome con un nivel muy alto de exigencia. Esta puesta al día constante, que cualquiera podría pensar que es lo normal en cualquier asignatura, requiere de un esfuerzo más importante de lo habitual en Prehistoria I dado que las novedades arqueológicas que se suceden mes tras mes acerca de qué pudo haber ocurrido en el Paleolítico marcan hitos y rupturas importantísimas con respecto a hipótesis y yacimientos ya conocidos. Al haber pocos datos para rellenar las lagunas existentes para este período, las novedades que se dan a conocer en revistas nacionales e internacionales tienen un impacto tremendo, de ahí que sea bastante frecuente encontrar publicaciones sobre este período en revista de reconocido prestigio mundial como Nature, Science, Plos One, etc. Esto no ocurre en absoluto en períodos cronológicos más recientes, donde los yacimientos excavados son más numerosos y las novedades no provocan cambios de paradigmas tan grandes.

Ciclos de Mejora en el Aula (2020). Experiencias de Innovación Docente de la US Esta obra se distribuye con la licencia Creative Commons 
Igualmente, empiezo a ganar experiencia para saber cuáles son los principales problemas y ventajas que suelen tener los alumnos y alumnas con cada tema para ir haciendo pequeñas modificaciones. Este año me he sentido por lo tanto más capacitado para ampliar el CIMA ya comenzado en cursos anteriores (Gómez Peña, 2018) a un número más elevado de clases. No obstante, la necesidad de realizar clases telemáticas ha generado dudas en mi manera de impartir el proyecto de innovación docente que comentaré posteriormente. Además, me sigue preocupando que se note mucho la diferente manera de impartir los contenidos entre los temas tratados mediante el CIMA y los dados de forma magistral. De hecho, el año pasado hubo una alumna que me lo comentó al terminar la asignatura.

Con respecto al alumnado al que se imparte la docencia de esta asignatura, es necesario aclarar que suele tener un conocimiento casi nulo de esta asignatura por dos motivos principales: por un lado, el escasísimo tratamiento de esta materia en secundaria y bachillerato, más volcado a impartir docencia de Historia Contemporánea. Por otra parte, la nula formación obtenida en la propia carrera por ser una de las primeras asignaturas que cursan. Ambos problemas podrían resultar anecdóticos si no fuera porque, tal y como revela un reciente estudio publicado (Gefaell et al., 2020), la mitad de los alumnos y alumnas universitarias (entre los que se ha encuestado a estudiantes de tercer curso de Biología, Química, Historia y Filología Inglesa de varias universidades españolas) no conoce cómo funciona la selección natural (mecanismo fundamental para la comprensión de la evolución de los organismos vivos, incluidos por supuesto los homininos, y que se analiza con relativa profundidad entre los temas 3 y 4 dentro de la asignatura).

Ciclos de Mejora en el Aula (2020). Experiencias de Innovación Docente de la US Esta obra se distribuye con la licencia Creative Commons 
A estos problemas formativos se une otro derivado del propio interés previo del alumnado hacia esta materia. Desde hace unos años, en la Facultad de Geografía e Historia se ha implantado un Grado en Arqueología donde suelen matricularse estudiantes que tienen un interés previo a su paso por las aulas por la arqueología en general y la prehistoria en particular. Esta situación ha derivado en que un elevado número de alumnos y alumnas que ingresan en el Grado de Historia lo haga porque tiene un interés previo en otros períodos o temáticas históricas.

Por otro lado, las circunstancias derivadas de la pandemia provocada por el coronavirus han afectado sensiblemente al modo en que suele impartirse la docencia de esta asignatura. Habitualmente, las clases se desarrollan en el Aula Magna de la Facultad de Geografía e Historia de la Universidad de Sevilla. Se trata de un espacio amplio, con capacidad suficiente para el elevado número de estudiantes que se matriculan en el primer curso del Grado en Historia, con una buena iluminación y la tecnología suficiente para llevar a cabo una docencia con buena acústica y buen apoyo visual. Sin embargo, debido a las medidas de seguridad y salud tomadas por el gobierno de la Junta de Andalucía se suspendieron las clases presenciales a partir del 10 de noviembre. Desde entonces, la docencia se realizó íntegramente de manera online a través de la aplicación Blackboard Collaborate Ultra, disponible desde la plataforma Enseñanza Virtual de la Universidad de Sevilla. Sobre estas circunstancias y sus consecuencias se hablará posteriormente a la hora de abordar cómo se ha aplicado el ciclo de innovación docente.

Ciclos de Mejora en el Aula (2020). Experiencias de Innovación Docente de la US Esta obra se distribuye con la licencia Creative Commons 


\section{Diseño previo del CIMA}

La asignatura Prehistoria I: Las sociedades cazadoras y recolectoras consta de nueve temas:

\section{BLOQUE I) INTRODUCCIÓN Y CORRIENTES DE PENSAMIENTO}

Tema 1: El concepto de Prehistoria

Tema 2: Corrientes epistemológicas en Prehistoria. Teorías, conceptos y métodos

\section{BLOQUE II) ECOSISTEMAS PREHISTÓRICOS}

Tema 3: Paleoecosistemas cuaternarios: El Pleistoceno

\section{BLOQUE III) GRUPOS CULTURALES DEL PLEISTOCENO}

Tema 4: Origen y evolución del ser humano

Tema 5: Organización económica y social de los cazadores-recolectores

Tema 6: Complejos tecnológicos de los grupos paleolíticos Tema 7: El mundo simbólico de los cazadores-recolectores

\section{BLOQUE IV) LOS ÚLTIMOS CAZADORES-RECOLECTORES}

Tema 8: Paleoecosistemas cuaternarios: El Holoceno Tema 9: Epipaleolíticos y mesolíticos

Teniendo presente que el número máximo de horas a impartir son sesenta, a cada tema le corresponde una media de 6-7 horas de clase. Por lo tanto, dado que el CIMA diseñado ha afectado a cuarenta horas, se planteó su realización dentro de los 6 primeros temas de la asignatura. En relación con lo acabado de comentar, la figura 1 muestra ordenados estructuralmente los temas que se han impartido en la asignatura, apareciendo en letras blancas aquéllos que se han visto afectados por el CIMA en su totalidad. Como se puede observar, se parte en los primeros temas de aspectos más generales a temas más específicos. Desde el método científico y sus particularidades en el estudio de la prehistoria, pasando por las diferencias 
corrientes epistemológicas. El tema 3 y el tema 8 son equivalentes a los tiempos Paleolítico y Mesolítico-Neolítico-Edad de los Metales respectivamente, por lo que los temas 4-7 y 9 están estrechamente vinculados a ambos.

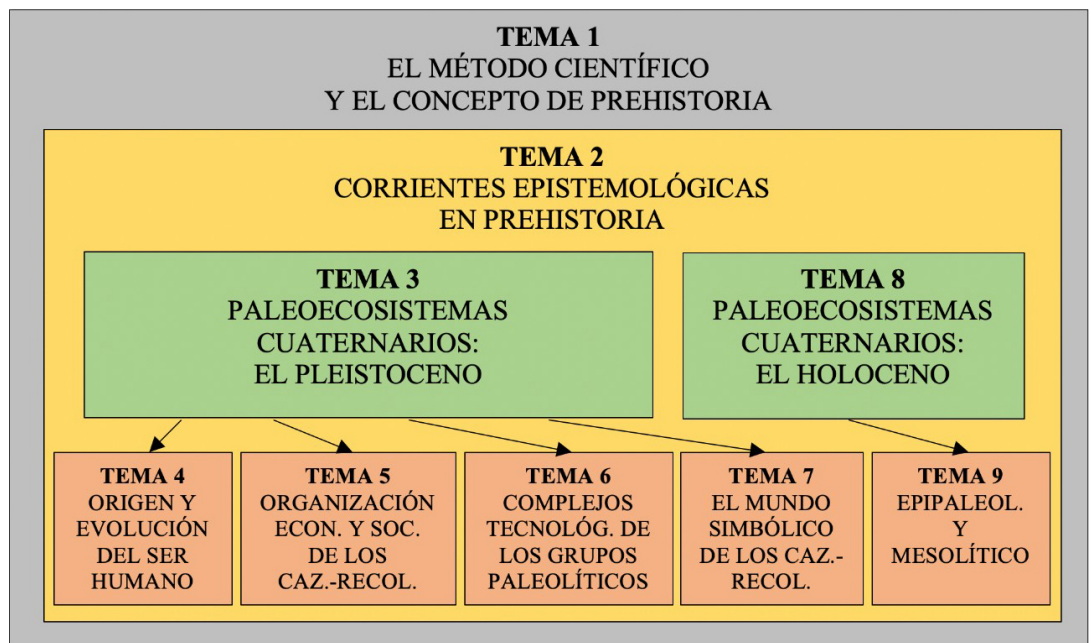

Figura 1. Estructura de contenidos de la asignatura de Prehistoria I (elaboración propia)

Se puede apreciar viendo la figura 1 que hay temas rectores que siempre van a aparecer periódicamente a lo largo del desarrollo de otros posteriores (1-2), tanto por los contenidos filosóficos como por los metodológicos explicados en ellos, mientras que hay otros temas (3-9) que presentan relaciones recíprocas. En este sentido, el marco paleoambiental del Pleistoceno y del Holoceno condicionan la evolución hominina y su cultural material, mientras que la propia acción de los homininos sobre su nicho ecológico condiciona las características de los biomas en lo que éstos viven. A esta relación mutua hacen referencia las flechas que conectan unos temas con otros. Además, en el desarrollo de este CIMA, dado que se tocan los cuatro niveles de desarrollo de la asignatura, se puede observar muy bien el efecto cascada que el aprendizaje de los primeros temas puede llegar a tener sobre temas posteriores (4-7 y 9). En este sentido ha sido muy interesante observar el 
nivel de aprendizaje de los estudiantes en las cuestiones anteriores a cada nuevo tema para ver cómo han ido modificando su visión y enriqueciendo las respuestas ofrecidas.

Tabla 1. Problemas a resolver por cada hora de clase del CIMA (elaboración propia)

\begin{tabular}{|c|c|c|c|c|c|}
\hline \multicolumn{6}{|c|}{ Tema 1. El método científico y el concepto de Prehistoria } \\
\hline $\begin{array}{l}\text { ¿Qué es la } \\
\text { Prehistoria? }\end{array}$ & $\begin{array}{l}\text { ¿Cómo se } \\
\text { obtienen } \\
\text { los datos en } \\
\text { Prehistoria? }\end{array}$ & $\begin{array}{c}\text { ¿La Prehistoria } \\
\text { forma parte de } \\
\text { la Historia? }\end{array}$ & $\begin{array}{c}\text { ¿La Prehistoria } \\
\text { es una } \\
\text { disciplina } \\
\text { científica? }\end{array}$ & $\begin{array}{c}\text { ¿Cómo se } \\
\text { hace ciencia? }\end{array}$ & $\begin{array}{c}\text { ¿Es posible } \\
\text { hacer ciencia } \\
\text { desde la } \\
\text { disciplina } \\
\text { prehistórica? } \\
\text { ¿Por qué? }\end{array}$ \\
\hline \multicolumn{6}{|c|}{ Tema 2. Conceptos epistemológicos en Prehistoria } \\
\hline $\begin{array}{l}\text { ¿Cuándo } \\
\text { surge la } \\
\text { disciplina } \\
\text { prehistórica? }\end{array}$ & $\begin{array}{l}\text { ¿Cómo surge } \\
\text { la disciplina } \\
\text { prehistórica? }\end{array}$ & $\begin{array}{c}\text { ¿Qué es la } \\
\text { epistemología? }\end{array}$ & $\begin{array}{c}\text { ¿Qué } \\
\text { corrientes } \\
\text { teóricas } \\
\text { y/o autores } \\
\text { conoces para } \\
\text { interpretar la } \\
\text { Prehistoria? }\end{array}$ & $\begin{array}{l}\text { ¿Por qué } \\
\text { cambian las } \\
\text { teorías en } \\
\text { Prehistoria? }\end{array}$ & $\begin{array}{c}\text { ¿Tiene sentido } \\
\text { la filosofía de } \\
\text { la Prehistoria? } \\
\text { ¿Por qué? }\end{array}$ \\
\hline \multicolumn{6}{|c|}{ Tema 3. Paleoecosistemas cuaternarios: el Pleistoceno } \\
\hline $\begin{array}{c}\text { ¿Qué es el } \\
\text { Pleistoceno? }\end{array}$ & $\begin{array}{c}\text { ¿Qué caracteriza } \\
\text { al Pleistoceno? }\end{array}$ & $\begin{array}{l}\text { ¿Existe el } \\
\text { cambio } \\
\text { climático? }\end{array}$ & $\begin{array}{l}\text { ¿Cómo } \\
\text { definirías } \\
\text { el cambio } \\
\text { climático? }\end{array}$ & $\begin{array}{l}\text { ¿Desde } \\
\text { cuándo } \\
\text { existe } \\
\text { cambio } \\
\text { climático? }\end{array}$ & $\begin{array}{l}\text { ¿La fauna y } \\
\text { la flora han } \\
\text { sido siempre } \\
\text { las mismas en } \\
\text { las mismas } \\
\text { regiones del } \\
\text { planeta? } \\
\text { ¿Por qué? }\end{array}$ \\
\hline \multicolumn{6}{|c|}{ Tema 4. Origen y evolución del ser humano } \\
\hline $\begin{array}{l}\text { ¿Cuándo } \\
\text { y dónde } \\
\text { surgió el ser } \\
\text { humano? }\end{array}$ & $\begin{array}{c}\text { ¿Sabrías } \\
\text { describir a un } \\
\text { Australopithecus } \\
\text { y nombrarme } \\
\text { algunas especies } \\
\text { de su género? }\end{array}$ & $\begin{array}{c}\text { ¿Sabrías } \\
\text { describir a un } \\
\text { Paranthropus } \\
\text { y nombrarme } \\
\text { algunas } \\
\text { especies de su } \\
\text { género? }\end{array}$ & $\begin{array}{c}\text { ¿Qué } \\
\text { características } \\
\text { anatómicas y } \\
\text { conductuales } \\
\text { forman parte } \\
\text { del género } \\
\text { Homo? }\end{array}$ & $\begin{array}{l}\text { ¿Cómo y por } \\
\text { qué surgen } \\
\text { las especies? }\end{array}$ & $\begin{array}{c}\text { ¿Los humanos } \\
\text { somos } \\
\text { diferentes } \\
\text { al resto de } \\
\text { especies } \\
\text { homininas? } \\
\text { ¿Por qué? }\end{array}$ \\
\hline
\end{tabular}

Ciclos de Mejora en el Aula (2020). Experiencias de Innovación Docente de la US Esta obra se distribuye con la licencia Creative Commons Reconocimiento-NoComercial-SinObraDerivada Internacional (CC BY-NC-ND 4.0.) 


\begin{tabular}{|c|c|c|c|c|c|}
\hline $\begin{array}{c}\text { ¿Es la } \\
\text { organización } \\
\text { familiar la } \\
\text { base del } \\
\text { Paleolítico? }\end{array}$ & $\begin{array}{l}\text { ¿Los cazadores- } \\
\text { recolectores } \\
\text { actuales nos } \\
\text { dan información } \\
\text { sobre el pasado? }\end{array}$ & $\begin{array}{l}\text { ¿Ha habido } \\
\text { otra forma } \\
\text { de economía } \\
\text { durante el } \\
\text { Paleolítico? }\end{array}$ & $\begin{array}{c}\text { ¿Qué } \\
\text { información } \\
\text { aportan } \\
\text { los restos } \\
\text { animales } \\
\text { sobre los } \\
\text { humanos del } \\
\text { Paleolítico? }\end{array}$ & $\begin{array}{c}\text { ¿Cómo } \\
\text { creció el } \\
\text { poblamiento } \\
\text { homínido? }\end{array}$ & $\begin{array}{l}\text { ¿Por qué no } \\
\text { somos ya } \\
\text { cazadores- } \\
\text { recolectores? }\end{array}$ \\
\hline \multicolumn{6}{|c|}{ Tema 6. Complejos tecnológicos de los grupos paleolíticos } \\
\hline $\begin{array}{c}\text { ¿Cómo se } \\
\text { identifica } \\
\text { la industria } \\
\text { lítica? }\end{array}$ & $\begin{array}{c}\text { ¿Qué es el Modo } \\
1 ?\end{array}$ & $\begin{array}{c}\text { ¿Qué es el } \\
\text { Modo 2? }\end{array}$ & $\begin{array}{l}\text { ¿Qué es el } \\
\text { Modo 3? }\end{array}$ & $\begin{array}{l}\text { ¿Qué es el } \\
\text { Modo 4? }\end{array}$ & $\begin{array}{c}\text { ¿Qué } \\
\text { importancia ha } \\
\text { tenido el uso } \\
\text { de industria } \\
\text { lítica en la } \\
\text { evolución } \\
\text { homínida? } \\
\text { ¿Por qué? }\end{array}$ \\
\hline
\end{tabular}

Centrándome en mi modelo ideal de docencia, éste se ha venido basando en los últimos años en un aprendizaje basado en la resolución de problemas para que los estudiantes vayan poco a poco cogiendo el hábito de trabajar y reflexionar en el aula, del mismo modo que tendrán que hacerlo posteriormente a la hora de desempeñar profesiones relacionadas con la Historia. Dentro de dicho modelo ideal, se previó empezar pasando un cuestionario a los y las estudiantes antes de comenzar cada tema para testar su nivel de conocimientos y ponerles en la pista sobre lo que se trataría en cada uno de ellos. Del mismo modo, se planeó pasar un segundo cuestionario tras finalizar cada tema con las mismas preguntas para ver el grado de conocimientos adquiridos. En cuanto al primer cuestionario, se subiría a la plataforma de Enseñanza Virtual la misma mañana en que se tuviera previsto comenzar un nuevo tema. Por lo que respecta al segundo, a la finalización de cada uno desde esa misma tarde también a través de Enseñanza Virtual. Las preguntas previstas de los cuestionarios prey post- han sido las recogidas en la tabla 1, mientras que el número de horas dedicado a cada tema y las fechas de realización de ambos cuestionarios aparecen en la tabla 2.

Ciclos de Mejora en el Aula (2020). Experiencias de Innovación Docente de la US Esta obra se distribuye con la licencia Creative Commons 
Tabla 2. Ordenación cronológica de los temas, duración en horas de cada uno, fechas de realización de los cuestionarios previos y posteriores, y fechas previsibles de impartición de cada uno de los temas (elaboración propia)

\begin{tabular}{|c|c|c|c|c|}
\hline Temas & Horas & $\begin{array}{c}\text { Realización del } \\
\text { cuestionario previo }\end{array}$ & $\begin{array}{c}\text { Fechas de inicio y } \\
\text { fin de cada tema }\end{array}$ & $\begin{array}{c}\text { Realización del } \\
\text { cuestionario posterior }\end{array}$ \\
\hline Tema 1 & 6 & 5 oct (mañana) & $5 /$ oct - 14/oct & 15 oct (mañana) \\
\hline Tema 2 & 8 & 15 oct (mañana) & $15 /$ oct - 28/oct & 29 oct (mañana) \\
\hline Tema 3 & 6 & 29 oct (mañana) & $29 /$ oct - 10/nov & 11 nov (mañana) \\
\hline Tema 4 & 7 & 11 nov (mañana) & $11 /$ nov - 23/nov & 23 nov (mañana) \\
\hline Tema 5 & 6 & 24 nov (mañana) & $24 /$ nov - 3/dic & 3 dic (mañana) \\
\hline Tema 6 & 7 & 4 dic (mañana) & $4 /$ dic - 21/dic & 22 dic (mañana) \\
\hline
\end{tabular}

Las sesiones de clase han comenzado otros años llevando a los estudiantes recursos bibliográficos sobre los aspectos a tratar en esa y posteriores sesiones para que durante dicha hora de clase quien quiera pueda consultar la obra, ver su índice, conocer la reseña del autor, su nombre, bibliografía relacionada con esa temática al final del libro, etc. Desafortunadamente, este último asunto no se ha podido aplicar durante este curso para minimizar en la medida de lo posible el intercambio de objetos entre los asistentes al aula. En su defecto, se previó recomendar los libros accediendo a su ficha a través del catálogo online de la Biblioteca de la Universidad de Sevilla. Los días que esto ocurriera, se le dedicaría a esta tarea no más de cinco minutos.

Tras estos primeros minutos de presentación de la obra, en el modelo ideal se pensó en proponer (tal y como se ha venido haciendo en cursos anteriores) una cuestión que tuviera relación con un problema teórico, metodológico o factual del temario de la asignatura con la intención de generar un primer debate entre los alumnos y alumnas.

Ciclos de Mejora en el Aula (2020). Experiencias de Innovación Docente de la US Esta obra se distribuye con la licencia Creative Commons 
No obstante, la pregunta tiene que relacionarse en la medida de lo posible con algún tema que despierte el interés y la curiosidad de los estudiantes para captar su atención (Thorndyke, 1977). Para ello se propone utilizar el conocido como modelo TORA, utilizado por Ham en la interpretación del patrimonio (Ham, 1992) y empleado en cursos anteriores como parte de innovaciones docentes previas (Gómez Peña, 2015). Bajo estas siglas se engloban explicaciones temáticas, organizadas, relevantes y atractivas. Con ello se pretende generar un debate con las ideas e hipótesis de los estudiantes y sus posibles argumentos. Mi labor en esta etapa de la sesión trataría por un lado de plantear nuevas preguntas menores para complejizar y hacer evolucionar el debate, y por otra parte apuntar y ordenar estructuralmente las ideas en la pizarra para realizar posteriores aclaraciones. La lección hasta este punto sirve para plantear posibles alternativas a un esquema preestablecido sobre este asunto que traigan previamente a clase los estudiantes (si es que lo tenían). Para ello es igualmente es importante tener presente el número de ideas a tratar en cada clase para no saturar a los estudiantes. Durante un tiempo pareció útil recurrir al llamado 'Principio del $7+/-2$ '. Este principio fue desarrollado por Miller (1956) y llama la atención sobre el número de ideas nuevas a las que de promedio los adultos jóvenes son capaces de memorizar a corto plazo la primera vez que las escuchan. En consecuencia, si estamos interesados en que todo el mundo capte nuestro mensaje al cien por cien el número de ideas principales debe ser siempre menor o igual que 5. Sin embargo, estudios posteriores (Cowan, 2001) recomiendan trabajar con un nuevo concepto, el del 'Número mágico 4', que limita las ideas principales a este valor para el mismo espectro de la sociedad. Por este motivo, en ninguna sesión de clase se pueden superar cuatro o cinco ideas principales. Sobre ellas deben pivotar las preguntas y respuestas, así como el debate posterior. A esta sección de la clase se le dedicarían veinticinco minutos.

Ciclos de Mejora en el Aula (2020). Experiencias de Innovación Docente de la US Esta obra se distribuye con la licencia Creative Commons 
No obstante, durante este curso académico me surgieron dos dudas importantes a la hora de poder aplicar el modelo ideal en contraposición al hipotético modelo real que preví antes de iniciar la docencia. En primer lugar, la asistencia a las sesiones se planteó dividida entre alumnos y alumnas presenciales y estudiantes telematizados. Por este motivo, pensé en probar a generar debates los primeros días y, en caso de que no fluyese el diálogo entre los estudiantes, trasladaría dichos debates a la plataforma de Enseñanza Virtual abriendo un foro de diálogo para que los estudiantes pudiesen expresarse con más tiempo, orden y tranquilidad.

En segundo término, habitualmente las nubes de ideas y los posteriores esquemas los realizo en la pizarra sobre la marcha. Creo que esto da frescura al ritmo de la clase y focaliza la atención de los estudiantes ante lo que se anota en ella, ya que por lo general suelen pensar que lo se deja apuntado en el aula es más importante que lo que simplemente se dice de viva voz, aunque solo sea por el simple hecho de pensar que quiero hacer que la idea perdure más y mejor en sus cabezas al preservarla por escrito. Sin embargo, este curso académico no se podría hacer esto si las cámaras que había que instalar en las aulas no grababan bien la pizarra con los brillos y las sombras de la iluminación del aula. Según saliera la prueba en las primeras horas de clase, esta idea habría que modificarla. Por ejemplo, dejando los esquemas previamente hechos en una diapositiva en el Power Point que solamente se mostrase tras el debate generado.

Avanzando en el desarrollo de la clase ideal, en una tercera fase dentro de la sesión de una hora habría lugar para reordenar las ideas ya visibles en la pizarra y empezar a profundizar en ellas. La estructura de contenidos de esta sección se basaría en explicar las diferentes hipótesis propuestas para dar respuesta a la pregunta, analizar los 
argumentos en que se basan, y en los casos en que la pregunta tenga estrecha relación con los datos arqueológicos mostrar a los estudiantes cuáles son esas piezas y contextos arqueológicos en que se basan las hipótesis. De nuevo, este último apartado de la clase tendría una duración también de veinticinco minutos.

\section{Aplicación del CIMA}

La aplicación del proyecto de innovación docente se ha llevado a cabo entre los días 5 de octubre y 22 de diciembre en un total de 40 horas lectivas. Sin lugar a dudas, esta labor ha estado marcada por las restricciones impuestas por la Junta de Andalucía y la Universidad de Sevilla. Desde el 5 de octubre hasta el 9 de noviembre las clases se llevaron a cabo de manera semipresencial, pasando desde esta fecha hasta el final del CIMA a ser completamente telemáticas. Esta circunstancia ha hecho necesario modificar algunos aspectos previstos en el diseño previo que se irán indicando a lo largo de este apartado cuando corresponda.

Como se dijo anteriormente, antes de dar comienzo a cada tema se ha informado por correo electrónico a los estudiantes que tenían disponible un cuestionario para su contestación y que en ningún caso dichas respuestas eran motivo de evaluación para la asignatura, por lo que podían responder con total tranquilidad siempre y cuando no buscasen información previamente. Debido a que los estudiantes han estado obligados desde el primer día a asistir a clases presencialmente dividiéndose en subgrupos que han ido rotando en las aulas semanalmente, desde un primer momento no ha sido posible pasar dichos cuestionarios en persona a los alumnos y alumnas para poder saber que no han consultado información sobre las preguntas propuestas antes de contestarlas.

Una vez en el aula, la propuesta de CIMA contemplaba la realización de sesiones monotemáticas de 60 minutos

Ciclos de Mejora en el Aula (2020). Experiencias de Innovación Docente de la US Esta obra se distribuye con la licencia Creative Commons 
en las cuales tratar diferentes subtemas englobados dentro del tema principal que se estuviera dando en cada momento. A propósito de este asunto, afortunadamente no ha habido grandes problemas. En la gran mayoría de las ocasiones se ha podido desarrollar cada una de las sesiones en su totalidad dentro del tiempo establecido. Tan solamente en tres ocasiones se redujo la clase a cincuenta minutos. La propuesta basada en resolución de problemas (problemas conceptuales y metodológicos en su aplicación al estudio teórico de la Prehistoria) se ha estructurado temporalmente en el aula del siguiente modo:

5' Minutos de cortesía para que terminasen de llegar los estudiantes, anoten sus nombres en los folios destinados a la trazabilidad del estudiantado presente en el aula por motivo del coronavirus y se instalen en clase dado que la asignatura es la primera que se imparte en el bloque de la tarde. En ese breve rato he aprovechado para firmar en Horfeus, abrir Enseñanza Virtual, abrir la presentación en Power Point dentro de la plataforma y comprobar que no hay problemas con el micrófono.

5' Por lo general, se ha comenzado recomendando bibliografía a los estudiantes sobre el tema a analizar durante esa hora de clase y durante las siguientes. Debido a la simultaneidad de clases presenciales y online, se ha recurrido a mostrar las referencias de las obras a través del buscador de la biblioteca de la Universidad de Sevilla para facilitar la labor a los estudiantes conectados desde casa. Otros cursos, los libros eran llevados a clase para fomentar la curiosidad y el interés por ellos, puesto que, entre otras cosas, las reseñas de las monografías recomendadas en clase han sido puntuables para subir nota (siempre y cuando se aprobase el examen al menos con un cinco). No obstante, su presentación de manera telemática no ha hecho que hayan mostrado menos interés a la hora de entregar un número de recensiones similar al del curso pasado. 
25' Posteriormente, se ha realizado un debate con los estudiantes planteándoles la pregunta recogida en la tabla 1. Para orientar las posteriores preguntas e ir extrayendo la información necesaria de los alumnos y alumnas que han participado en el debate, previamente se habían analizado las respuestas dadas en el cuestionario al comienzo del tema. Teniendo presentes los errores, dudas y ausencias en dichas respuestas, orientar el debate y las cuestiones realizando segundas y terceras preguntas era más sencillo. La intervención de los estudiantes a través de la aportación de ideas y dudas ha generado habitualmente una cascada de conceptos y una retroalimentación entre ellos. Tras ello, se ha realizado por regla general una primera aclaración de conceptos tras el debate realizando una primera estructuración básica de los mismos. Para ello, se ha incentivado a los estudiantes presenciales a que llevasen sus portátiles, tablets y móviles a clase para que pudieran seguir las intervenciones del resto de compañeros y compañeras tanto por micrófono como por el chat de Enseñanza Virtual.

En este punto de la clase es donde han ocurrido los problemas más importantes en relación con los planificado en el modelo ideal. El más importante de ellos ha estado intimamente relacionado con la necesidad de realizar debates de manera telemática con los estudiantes que no podían asistir al aula. Esto generó dos inconvenientes en el desarrollo de la docencia. El primero, que los alumnos y alumnas conectados a Blackboard Collaborate Ultra no escuchaban a los compañeros y compañeras que debatían desde la clase. Esto hizo que tuviera que repetir y resumir constantemente las ideas aportadas por estos últimos a través del micrófono para integrar a todos y todas constantemente en esta parte de la docencia. El segundo vino derivado por la necesidad de impartir las clases de manera exclusivamente telemática desde el 10 de noviembre. A partir de esta fecha, muchos estudiantes dejaron de participar. Los motivos dados eran varios: no tener micrófono, tener malas conexiones que hacía que entrasen y saliesen frecuentemente de Enseñanza Virtual, no 
encontrarse solos y en silencio en la habitación en la que se encontraban recibiendo la docencia, tener problemas por la deficiencia de sus conexiones para escuchar a otros compañeros o al profesor sin que se entrecortara el sonido, etc.; por todo ello, otra de las mayores dificultades la he encontrado a la hora de respetar los tiempos previstos. En unos casos porque los debates se han alargado más del tiempo previsto (tanto por lo motivos acabados de exponer como por la presencia de alumnos del aula de la experiencia que ha facilitado dinamizar las participaciones) y en otras ocasiones porque los estudiantes tenían más claras de lo que pensaba algunas cuestiones (lo que ha permitido profundizar más en algunas partes del temario y enriquecer con más datos e hipótesis los asuntos a tratar).

25' Profundización en los principales conceptos antes introducidos en el debate tras su estructuración y digestión entre la pizarra y el Power Point en Enseñanza Virtual con un análisis profundo de las hipótesis y datos debatidos previamente para ver sus puntos fuertes y débiles.

\section{Evaluación del CIMA}

El sistema de evaluación del CIMA durante este curso 2020/2021 ha sido el mismo de cursos anteriores: la elaboración de un cuestionario de manera previa y posterior a la impartición de la materia. En relación con este asunto, ya expresé en una anterior publicación que era necesario comparar los resultados obtenidos en este tipo de innovación docente con los obtenidos en clases impartidas a otros grupos de la misma asignatura que no hubiesen recibido dicha metodología de innovación docente con la intención de poder concluir que dicho proyecto docente mejora el aprendizaje de los estudiantes (Gómez Peña 2018: 460).

Afortunadamente, lo acabado de indicar ha podido solventarse en el presente curso académico dado que ha sido posible comparar la idoneidad de mi CIMA. aplicado

Ciclos de Mejora en el Aula (2020). Experiencias de Innovación Docente de la US Esta obra se distribuye con la licencia Creative Commons 
a los estudiantes del Grupo 3 de Prehistoria I, con los resultados de la escalera de aprendizaje de los estudiantes del Grupo 1 de la misma asignatura, a quienes se ha impartido docencia siguiendo exclusivamente el modelo de clase magistral. Para ello, a estos últimos se les ha pasado el cuestionario previo y el final con las mismas preguntas que al alumnado del Grupo 3. La comparativa de ambas escaleras mostrará el éxito o no de dicha metodología basada en la resolución de problemas.

Igualmente, entre las diferencias metodológicas de la docencia que se ha impartido a los Grupos 1 y 3 cabe mencionar aquí el número de ideas sobre las que ha pivotado cada hora de clase. Mientras que con el Grupo del CIMA se ha partido de preguntas y problemas cercanos al interés y conocimiento de los estudiantes, con el Grupo 1 se han explicado los temas siguiendo los esquemas e ideas básicos que habitualmente se encuentran presentes en los manuales. Asimismo, mientras que en la docencia del Grupo 3 se han tratado exclusivamente cuatro ideas principales por sesión sobre las que ha girado su aprendizaje para afianzar conceptos clave y no saturar de información a los estudiantes, con los discentes del Grupo 1 no se ha tenido en cuenta este número máximo, surgiendo en el aula tantas ideas como ha sido necesario durante la explicación de los contenidos de la asignatura.

Tabla 3. Número de estudiantes que ha contestado en cada tema a ambos cuestionarios (elaboración propia)

\begin{tabular}{|c|c|c|}
\hline Temas & $\begin{array}{c}\text { Grupo 1 (clase magistral) } \\
\text { Número total de estudiantes: 78 }\end{array}$ & $\begin{array}{c}\text { Grupo 3 (CIMA) } \\
\text { Número total de estudiantes: 50 }\end{array}$ \\
\hline Tema 1 & 75 & 48 \\
\hline Tema 2 & 72 & 47 \\
\hline Tema 3 & 73 & 47 \\
\hline Tema 4 & 71 & 48 \\
\hline Tema 5 & 68 & 46 \\
\hline Tema 6 & 71 & 45 \\
\hline
\end{tabular}

Ciclos de Mejora en el Aula (2020). Experiencias de Innovación Docente de la US Esta obra se distribuye con la licencia Creative Commons 
Pasando a hablar de los resultados obtenidos, el proceso de evaluación de los conocimientos iniciales y finales se ha realizado en el Grupo 3 sobre un total de 50 estudiantes, haciéndose lo propio con respecto al Grupo 1 sobre un máximo de 78 alumnos y alumnas matriculados. No obstante, no todos los estudiantes en cada caso han respondido a los cuestionarios previo y post, por lo que los cálculos hay que hacerlos teniendo en cuenta los siguientes números máximos de alumnos y alumnos en cada tema recogido en la tabla 3, cuestión que deja los resultados reflejados en las figuras 2 a 7.

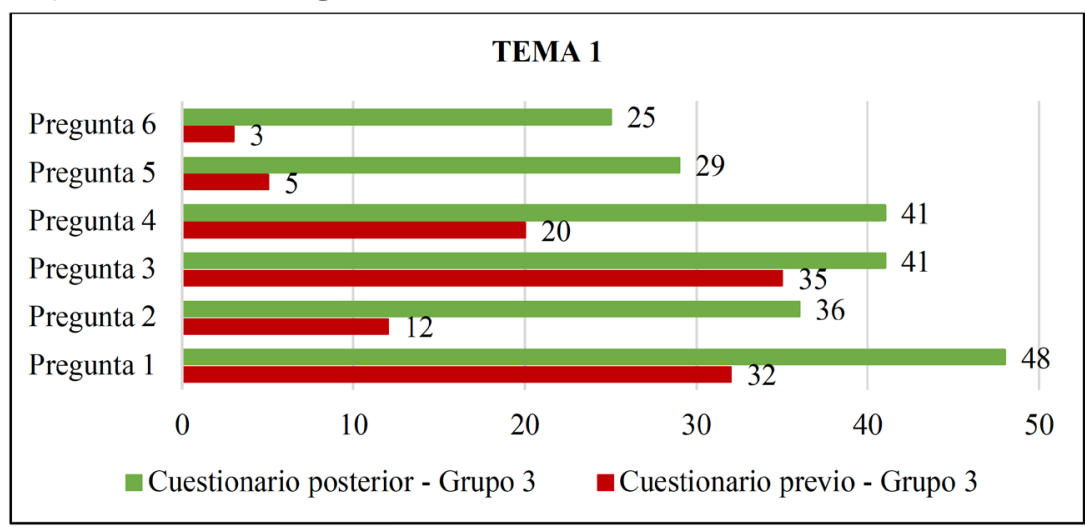

Figura 2. Gráfica con los resultados de las preguntas de la tabla 1 recogidos en los cuestionarios previo y posterior del Tema 1 (elaboración propia)

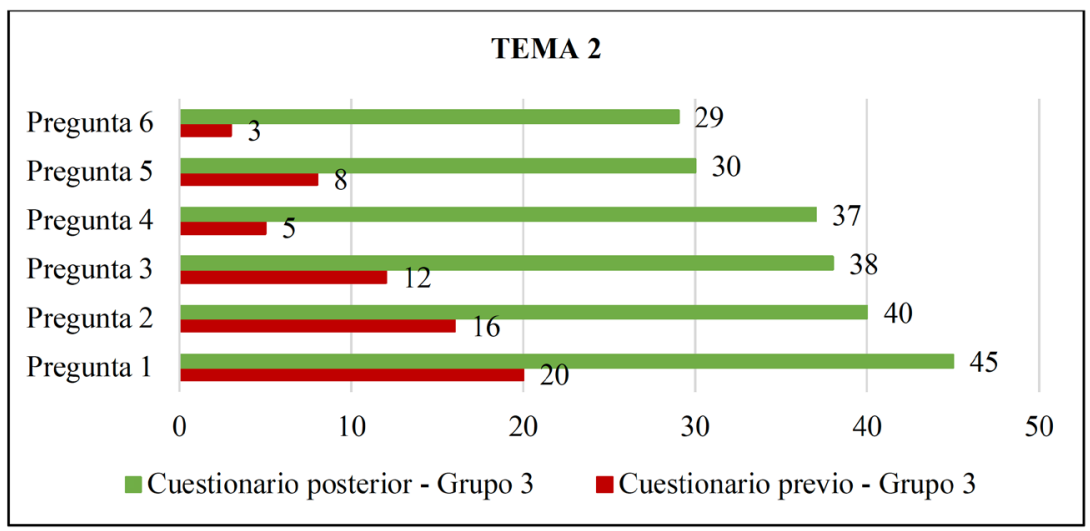

Figura 3. Gráfica con los resultados de las preguntas de la tabla 1 recogidos en los cuestionarios previo y posterior del Tema 2 (elaboración propia) Ciclos de Mejora en el Aula (2020). Experiencias de Innovación Docente de la US Reconocimiento-NoComercial-SinObraDerivada $\quad 4.0$ Internacional (CC BY-NC-ND 4.0.) 
TEMA 3

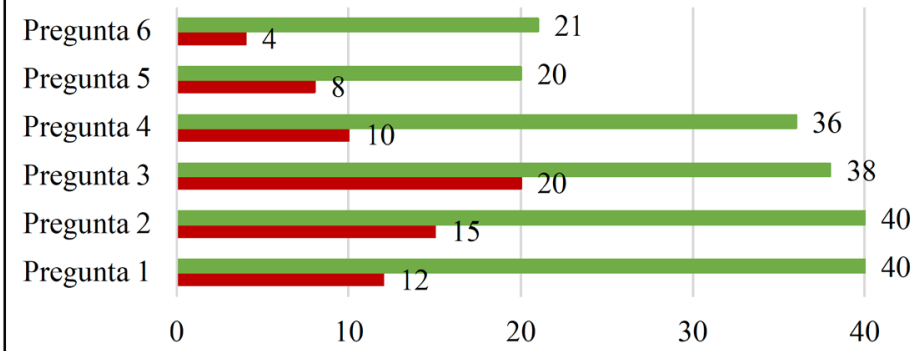

Cuestionario posterior - Grupo 3 Cuestionario previo - Grupo 3

Figura 4. Gráfica con los resultados de las preguntas de la tabla 1 recogidos en los cuestionarios previo y posterior del Tema 3 (elaboración propia)

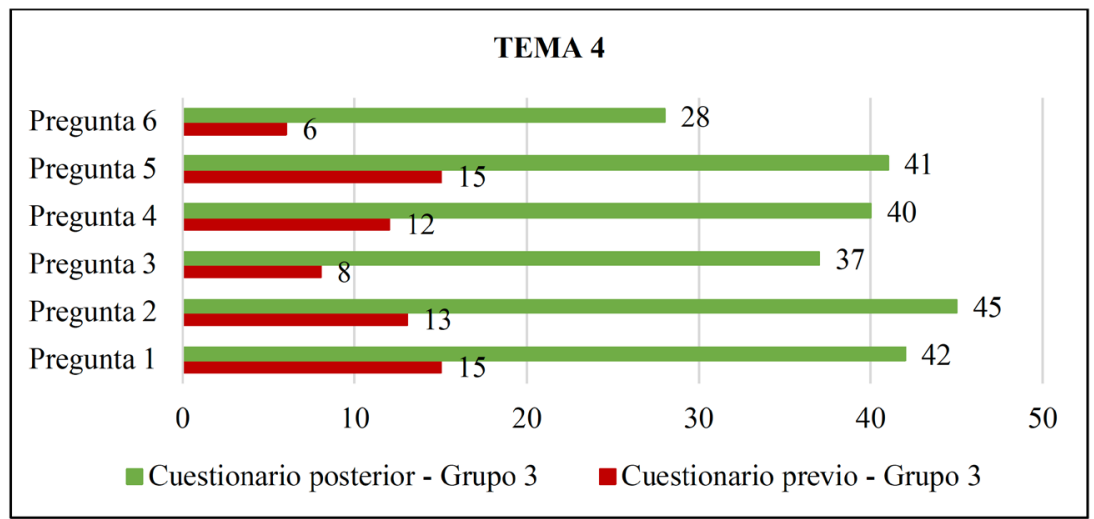

Figura 5. Gráfica con los resultados de las preguntas de la tabla 1 recogidos en los cuestionarios previo y posterior del Tema 4 (elaboración propia)

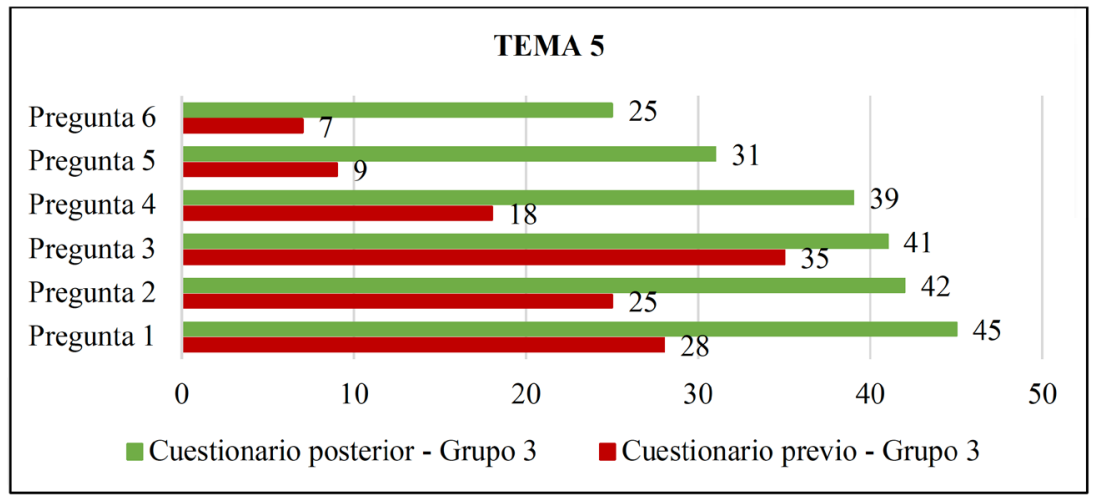

Figura 6. Gráfica con los resultados de las preguntas de la tabla 1 recogidos en los cuestionarios previo y posterior del Tema 5 (elaboración propia)

Ciclos de Mejora en el Aula (2020). Experiencias de Innovación Docente de la US (c) Esta obra se distribuye con la licencia Creative Commons Reconocimiento-NoComercial-SinObraDerivada $\quad 4.0$ Internacional (CC BY-NC-ND 4.0.) 


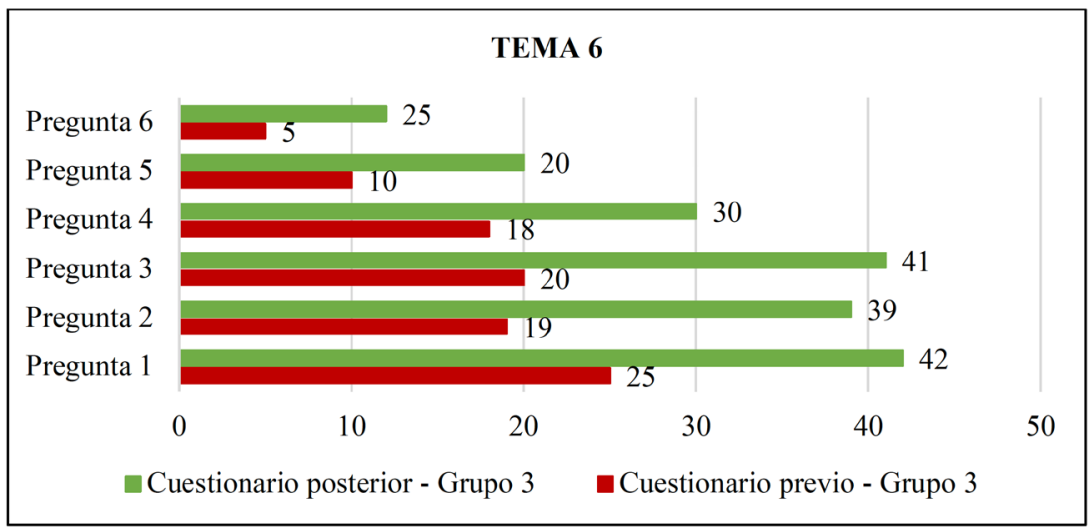

Figura 7. Gráfica con los resultados de las preguntas de la tabla 1 recogidos en los cuestionarios previo y posterior del Tema 6 (elaboración propia)

Los resultados de los cuestionarios posteriores a la conclusión de los diversos temas dentro del Grupo 3 revelan los siguientes datos en comparación con los registrados dentro del Grupo 1, tal y como se muestra en la tabla 4.

A la vista de estos porcentajes, se puede afirmar tras realizar esta experiencia docente aplicada a los Grupos 1 y 3 a los que se les ha impartido el mismo temario dentro de la asignatura de Prehistoria I: las sociedades cazadoras y recolectoras que el modelo de innovación basado en la resolución de problemas afecta positivamente al aprendizaje de los estudiantes en un 9,1\% más de casos.

Tabla 4. Porcentaje de éxito en las respuestas a los cuestionarios posteriores entre los Grupos 1 y3 (elaboración propia)

\begin{tabular}{|c|c|c|}
\hline Temas & $\begin{array}{c}\text { Porcentaje de éxito del } \\
\text { grupo 1 (clase magistral) }\end{array}$ & $\begin{array}{c}\text { Porcentaje de éxito del } \\
\text { grupo 3 (CIMA) }\end{array}$ \\
\hline Tema 1 & $67,25 \%$ & $76,38 \%$ \\
\hline Tema 2 & $66,48 \%$ & $77,65 \%$ \\
\hline Tema 3 & $60,53 \%$ & $69,14 \%$ \\
\hline
\end{tabular}

Ciclos de Mejora en el Aula (2020). Experiencias de Innovación Docente de la US Esta obra se distribuye con la licencia Creative Commons 


\begin{tabular}{|c|c|c|}
\hline Tema 4 & $71,22 \%$ & $80,90 \%$ \\
\hline Tema 5 & $42,32 \%$ & $51,71 \%$ \\
\hline Tema 6 & $41,23 \%$ & $47,84 \%$ \\
\hline
\end{tabular}

Igualmente, es oportuno realizar en este apartado final algunas reflexiones sobre las posibilidades de cambio y mejora para continuar aplicando con mayor éxito esta metodología de innovación docente en cursos venideros:

(1) Dudo sobre si hacer obligatorias (pero en ningún caso evaluables) las respuestas a los cuestionarios previo y posterior para maximizar el número de casos evaluables.

(2) Valoro integrar los temas 4, 5, 6 y 7 en un gran capítulo dentro de la asignatura. Hasta el momento, los contenidos relacionados con estos bloques dividen la materia por temas, analizándose cada uno de ellos de manera cronológica (evolución anatómica primero, evolución tecnológica después, evolución socio-económica en tercer lugar y por último evolución de las creencias religiosas). Este encorsetamiento que impone el programa de estudios hace que muchos estudiantes se sientan en ocasiones confusos a la hora de sincronizar en el tiempo todas las novedades somáticas y culturales vistas en los diferentes temas. Y, de hecho, considero que este es el gran problema de la asignatura en estos momentos. Un nuevo ordenamiento de los contenidos que permita impartir todas estas cuestiones a la vez desde un punto de vista cronológico sería un reto importante de mejora para un próximo CIMA.

(3) En caso de aplicar los cambios del punto anterior, sería necesario reestructurar las preguntas de los cuestionarios previo y posterior que afectan a dichos temas. Igualmente habría que valorar si los porcentajes de éxito en las respuestas a dichos cuestionarios arrojan mejores o peores resultados que los publicados en este estudio, 
lo que permitiría empezar a aceptar o desestimar dicha reestructuración.

(4) Por último, queda por saber si el cuestionario de este curso hubiera tenido porcentajes de éxito diferentes si los grupos 1 y 3 hubieran recibido exclusivamente docencia presencial y no también telemática. En este sentido, me planteo como duda acerca de la conveniencia o no de comparar los resultados de futuros años académicos con los de este curso 2020/2021.

Ciclos de Mejora en el Aula (2020). Experiencias de Innovación Docente de la US Esta obra se distribuye con la licencia Creative Commons 
Palabras clave: Prehistoria I, Grado en Historia, Docencia universitaria, Experimentación docente universitaria, Resolución de problemas

Keywords: Prehistory I, Degree in History, University Teaching, University Teaching Experimentation, Problem Resolution

\section{Referencias bibliográficas}

Cowan, N. (2001). The magical number 4 in short-term memory: A reconsideration of mental storage capacity. Behavioral and Brain Sciences, 24 (1), 87-114.

Gefaell, J., Prieto, T., Abdelaziz, M., Álvarez, I., Antón, J., Arroyo, J., Bella, J.L., Botella, M., Bugallo, A., Claramonte, V., Gijón, J., Lizarte, E., Maroto, R.M., Megías, M., Milá, B., Ramón, C., Vila, M. y Rolán-Alvarez, E. (2020). Acceptance and knowledge of evolutionary theory among third-year university students in Spain. PLOS ONE 15 (9): e0238345. https://doi.org/10.1371/journal.pone.0238345

Gómez Peña, A. (2015). El modelo TORA en las aulas: el caso de las espadas del Bronce Final halladas en la Península Ibérica. En R. Porlán y. E. Navarro (Coords.), II Jornadas de Docencia Universitaria. Universidad de Sevilla (2 y 3 de julio de 2015) (pp. 183-194). Sevilla: Universidad de Sevilla.

Gómez Peña, A. (2018). Un ciclo de mejora docente basado en la resolución de problemas aplicado a la asignatura de Prehistoria I. En R. Porlán y. E. Navarro (Coords.), Jornadas de Formación e Innovación Docente del Profesorado. Universidad de Sevilla (20 y 21 de diciembre de 2018) (pp. 450-465). Sevilla: Universidad de Sevilla. http://dx.doi.org/10.12795/JDU.2018.i01.25

Ham, S. (1992). Environmental interpretation: A practical guide for people with big ideas and small budgets. Golden: North American Press.

Miller, G.A. (1956). The magical number seven, plus or minus two: Some limits on our capacity for processing information. Psychological Review, 63 (2), 81-97.

Porlán Ariza, R. (coord.) (2017). Enseñanza Universitaria. Cómo mejorarla. Madrid: Ediciones Morata.

Thorndyke, P.W. (1977). Cognitive Structures in Comprehension and Memory of Narrative Discurse. Cognitive Psychology, 9 (1), 77-110. Ciclos de Mejora en el Aula (2020). Experiencias de Innovación Docente de la US 